livraisons

d'Histoire

de l'Architecture

\section{Livraisons de l'histoire de l'architecture}

13 | 2007

Architectures des établissements d'enseignement supérieur

\title{
Un programme novateur : L'Institut de paléontologie humaine d'Emmanuel Pontremoli
}

An innovative program : the Institute for Human Paleontology of Emmanuel Pontremoli

Ein innovatives Bauprogramm: das Institut de Paléontologie humaine

Pontremolis

\section{Arnaud Hurel et Alain Dubourg}

\section{OpenEdition \\ Journals}

Édition électronique

URL : http://journals.openedition.org/lha/403

DOI : $10.4000 /$ /ha. 403

ISSN : 1960-5994

Éditeur

Association Livraisons d'histoire de l'architecture - LHA

Édition imprimée

Date de publication : 10 juin 2007

Pagination : 51-64

ISSN : 1627-4970

Référence électronique

Arnaud Hurel et Alain Dubourg, « Un programme novateur : L'Institut de paléontologie

humaine d'Emmanuel Pontremoli », Livraisons de l'histoire de l'architecture [En ligne], 13 | 2007, mis en ligne le 10 juin 2009, consulté le 01 mai 2019. URL : http://journals.openedition.org//ha/403 ; DOI : $10.4000 /$ lha.403

Ce document a été généré automatiquement le 1 mai 2019.

Tous droits réservés à l'Association LHA 


\title{
Un programme novateur : L'Institut de paléontologie humaine d'Emmanuel Pontremoli
}

\author{
An innovative program : the Institute for Human Paleontology of Emmanuel \\ Pontremoli \\ Ein innovatives Bauprogramm: das Institut de Paléontologie humaine \\ Pontremolis
}

Arnaud Hurel et Alain Dubourg

$1 \mathrm{Au}$ début $\mathrm{du} \mathrm{XX}^{\mathrm{e}}$ siècle, les préhistoriens français forment encore une communauté disparate composée essentiellement d'amateurs qui œuvrent librement et isolément et qui, à défaut de reconnaissance de leurs études par l'Université, n'ont alors que les sociétés savantes et les congrès, internationaux (à partir de 1867) puis nationaux (1905), pour se former et confronter leurs travaux. L'initiative du prince Albert $\mathrm{I}^{\mathrm{er}} \mathrm{de}$ Monaco (1848-1922) de créer en 1910 l'Institut de paléontologie humaine (I.P.H.) marque de ce point de vue une rupture, car elle représente une étape importante dans l'institutionnalisation des sciences préhistoriques, c'est-à-dire dans la transformation d'une réalité sociale en une structure régulée.

2 Ses professeurs ${ }^{1}$ vont disposer des moyens leur permettant de mener des travaux de manière pérenne, le centre de recherches prenant en charge l'intégralité des moyens nécessaires à la production du savoir. Son succès va être immédiat et, au fil des années, il va attirer des chercheurs et des étudiants de nombreux pays étrangers, mais également financer ou mener pour son propre compte des fouilles majeures. Avant la première guerre mondiale, il se révèle sans équivalent au monde, y compris aux États-Unis où la pratique du mécénat scientifique est pourtant ancienne. Neuf dans son objet, « le progrès de la Science sur toutes les questions relatives à l'origine et à l'histoire de l'homme fossile » (c'est-à-dire à la fois l'étude des caractères et évolutions physiques, mais également les comportements culturels et cultuels), il l'est aussi dans son mode de 
fonctionnement (concentration en une institution unique de l'ensemble des moyens d'études et de valorisation des recherches).

Pour servir cette ambition, le Prince va commander à un architecte reconnu, Emmanuel Pontremoli (1865-1956), un bâtiment pratique, raffiné, inspiré par les orientations scientifiques de l'institut et représentatif d'une période de transition entre éclectisme et mouvement moderne.

\section{Concevoir un établissement scientifique d'un type nouveau}

\section{Mécénat scientifique}

4 Albert $\mathrm{I}^{\mathrm{er}}$ est un véritable savant dans le domaine de l'océanographie. Dans celui de l'archéologie préhistorique, même s'il a lui-même initié ou encouragé des fouilles (en particulier dans les grottes de Grimaldi), son rôle a été plus institutionnel, plus proche de celui d'un mécène (musée d'anthropologie préhistorique de Monaco, contrats d'édition et de recherche au profit de l'abbé Breuil, etc.). Pour autant, avec l'I.P.H. il n'entend pas limiter son soutien à une aide ponctuelle, aussi généreuse soit-elle. Comme pour l'Institut océanographique, il l'inscrit dans une perspective plus pérenne et donc plus ambitieuse. Il vise à mettre en place une structure apte à assumer l'intégralité du travail, de la fouille à la publication.

5 À l'automne 1909, il charge l'abbé Breuil et Marcellin Boule (1861-1942), le professeur de paléontologie du Muséum, d'en préparer le projet scientifique dont le socle réside dans une complémentarité avec les institutions scientifiques officielles françaises et, néanmoins, l'anticipation des moyens nécessaires à la préservation de l'indépendance institutionnelle. Il choisit à cet effet la forme juridique de la fondation, pour laquelle il obtient tout de suite la reconnaissance d'utilité publique, la dote d'un budget conséquent et d'un bâtiment pour abriter ses activités.

Le Prince s'implique directement dans la conception de l'édifice qui représente pour lui une traduction dans la pierre de ses idéaux nourris d'un messianisme scientifique et technique au service de l'émergence d'une conscience universelle. L'inauguration des conférences de l'Institut, le 18 février 1922, sera d'ailleurs pour lui l'occasion d'user du vocabulaire empreint de religiosité propre au scientisme :

Vous êtes ici dans un temple nouveau que j'ai fait sortir de terre pour que l'anthropologie, appuyée sur des lois solennelles, puisse planer un jour sur les mystères qui nous enveloppent. Je veux qu'elle apporte à la civilisation le concours des grandes forces contenues dans son sein et qui purifieront nos mœurs, nos idées, nos rapports sociaux, quand l'Humanité saura d'où elle vient et comprendra où elle va.

\section{Le choix d'un terrain}

7 Voulant marquer la proximité scientifique entre la nouvelle institution et le Muséum, et favoriser ainsi la constitution d'un pôle scientifique unique en son genre, les initiateurs du projet ont prospecté des terrains à proximité du Jardin des Plantes. Une implantation rue Cuvier est un temps envisagée, mais le choix se porte sur deux parcelles du lotissement de l'ancien marché aux chevaux où la Ville de Paris, qui vient de décider de réhabiliter ce quartier, fait percer des rues nouvelles. Le Prince souhaite acquérir des lots 
placés de part et d'autre de l'une de ces voies (aujourd'hui la rue René Panhard, perpendiculaire au boulevard St-Marcel), pour y bâtir d'un côté l'I.P.H. et de l'autre sa résidence parisienne.

Le principe d'une vente de gré à gré est repoussé par le conseil municipal de Paris au motif que le projet scientifique du Prince «n'a rien de proprement municipal» et que l'estimation de la valeur des lots est ancienne. Le principe d'une vente par adjudication est imposé. Le 11 juillet 1911, le Prince acquiert au profit de la Fondation, pour un montant total de 200000 francs (680 000 euros de 2006), un terrain d'un seul tenant d'une superficie notablement plus étendue (environ $1200 \mathrm{~m}^{2}$ ) que celui dont la cession avait été à l'origine demandée à la Ville.

Début novembre 1911, les premières entreprises de construction se mettent au travail et, à l'été, la maçonnerie est déjà très avancée. En juin 1913 le Prince envisage même une inauguration à l'automne. Si celle-ci ne se tient pas à cette époque, la fin de l'année permet toutefois de prendre possession des lieux ${ }^{2}$ et, le 29 décembre, le conseil d'administration peut s'y réunir pour la première fois.

10 À cette date, le gros œuvre est achevé et les services administratifs, les cabinets des professeurs et quelques salles (dessin, laboratoires de photographie et de chimie) sur le point de l'être. Les premières collections sont installées dans les salles de comparaison. Le mois de novembre 1914 est choisi pour l'inauguration officielle, le début des cours et une petite série de conférences destinées au grand public. Mais la première guerre mondiale va bouleverser tous les plans et ce n'est que le 23 décembre 1920 que l'institut sera inauguré en présence du Président de la République et des plus hautes autorités politiques et universitaires.

\section{Deux grands prix de Rome pour un institut de recherche}

\section{Emmanuel Pontremoli}

11 Le parcours professionnel de Pontremoli, qui allie architecture pure et archéologie, le prédisposait à la réalisation d'un édifice consacré à la science ${ }^{3}$. Après son baccalauréat ès sciences, il intègre l'École des arts décoratifs de Nice (1882) où il prépare le concours d'entrée à l'École des beaux-arts de Paris, qu'il intègre en 1883 dans l'atelier de LouisJules André (1819-1890). Au début de l'année 1890, il obtient le prix Rougevin, prix de composition décorative et se classe deuxième du concours de sortie, dont le sujet proposé était d'un esprit proche de la commande que lui passera le Prince :

Quelque chose d'assez inédit, sortant tout à fait des programmes habituels et trop rebattus, un édifice pratique, industriel en somme: le magasin des décors d'un grand théâtre, avec les ateliers où se fabriquent et se peignent toiles et portants.

12 Ce travail et celui du concours définitif, une allégorie dédiée à Jeanne d'Arc, sont remarqués par Charles Garnier (1825-1898) et magistralement récompensés par le premier Grand prix de Rome. Pensionnaire de l'Académie de France à Rome (1891-1896), il voyage beaucoup sur tout le pourtour méditerranéen. Après avoir travaillé à Pergame, c'est en tant qu'architecte et photographe qu'il participe aux fouilles du sanctuaire de l'Apollon de Didymes (1896-1897) sous la direction de Bernard Haussoullier (1853-1926).

De retour à Paris, Pontremoli est nommé inspecteur des bâtiments civils au musée du Louvre. À ce titre, il assiste Gaston Redon (1853-1956). Il est également chargé de dresser 
les plans et d'exécuter le « Palais de la femme » de l'exposition universelle de 1900 puis, en 1901, est nommé architecte en chef de l'observatoire de Paris. Gravement malade, il part pour la Côte d'Azur où il passe sa convalescence à Beaulieu, « le plus bel endroit du monde $»^{5}$. L'helléniste Théodore Reinach (1860-1928) y a acheté un éperon rocheux, la « Pointe Fourmi ». Il lui confie son désir d'y faire construire « une maison grecque » (qui deviendra la villa Kérylos), proposition qui enthousiasme l'architecte :

Je savais, et les expériences déjà tentées me le faisaient amplement sentir, que toute restauration, reproduction, reconstitution d'une demeure du passé est vide de sens si on s'attache exclusivement à ce qu'on croit être la vérité, ou la prétendue vérité archéologique. Je savais aussi que cette recherche est vaine, vouée au plus irrémédiable échec, puisque dès les premières tentatives le document exact, probant, ferait défaut et que dès lors tout s'évanouirait par manque de bases certaines; l'œuvre ainsi conçue ne pourrait être qu'un décor sans vie, le jouet d'un moment, la curiosité d'une heure ${ }^{6}$.

Pour imaginer et réaliser l'édifice commandé, une maison "vraie » et "actuelle ", Pontremoli bénéficie de la fortune personnelle des époux Reinach. Il peut ainsi rassembler autour de lui, entre 1903 et 1911, ses amis artistes de Rome et les plus grands artisans parisiens ou locaux. "Avec eux rien d'impossible et, s'il le fallait, on recommençait pour parvenir au résultat désiré $»^{7}$ : bas-reliefs en stuc exécutés par Paul Gasq (1860-1944), mobilier réalisé sur les dessins de Pontremoli par l'ébéniste Bettenfeld, fresques peintes et mosaïques de Gustave Jaulmes (1873-1959) et Adrien Karbowsky (1855-1945). Outre la réalisation de cette commande privée, il poursuit son travail pour l'État et est nommé, en 1904, architecte en chef du Muséum. Quittant l'observatoire, il abandonne les « étoiles pour la création tout entière ${ }^{8}$.

Pontremoli établit de multiples projets pour y rénover les bâtiments car «à l'histoire des animaux, s'ajoute, dans cet endroit, celle de toutes les architectures depuis Louis XIII jusqu'à nos jours. " ${ }^{9}$ Pendant les dix-sept années de ses fonctions, il va moderniser plusieurs édifices, mais également réaliser des laboratoires, une nouvelle Orangerie et créer le vivarium de la ménagerie.

\section{Constant Roux}

Pour l'I.P.H., Pontremoli veut offrir à la sculpture une place originale et non accessoire : exprimer la personnalité et la vocation scientifique du lieu. Par son architecture originale, le bâtiment attirera l'œil du passant, mais c'est sa frise sculptée, qui court sur toutes les façades au-dessus du soubassement, œuvre de Constant Roux (1865-1942), qui le retiendra.

La formation de Roux a débuté à l'École des beaux-arts de Marseille. " Monté » à Paris en 1884, il intègre les Beaux-Arts en 1888 et l'atelier de Jules Cavelier (1814-1894). En 1894, le concours du Prix de Rome ("Achille revêtant l'armure apportée par Thétis, sa mère ») lui permet d'obtenir le prix Maubert et de devenir pensionnaire de la Villa Médicis. À son retour à Marseille, en 1899, l'armateur Jules Charles-Roux (1841-1918) lui ouvre les portes d'une gloire locale, qui ne tarde pas à s'étendre à Paris.

Bien qu'auteur de nombreux travaux figurant en bonne place dans le panthéon des commandes publiques (comme la "République» et le monument à Antoine-Fortuné Marion au Palais Longchamp à Marseille, le "Souvenir des députés morts pour la France » du Palais Bourbon), Roux n'a pas laissé le même souvenir artistique que Pontremoli. Il est 
vrai que son art, reconnu, est d'un classicisme conforme au goût de l'époque et, partant, n'émerge pas.

19 Ses créations ont accompagné les choix et les passions scientifiques du prince de Monaco ${ }^{10}$ : lustres pour le musée océanographique (1908), épée d'académicien d'Albert ${ }^{\text {er }}$ à l'occasion de sa seconde élection à l'Académie des sciences (1909), armoiries de la famille Grimaldi à l'entrée de l'Institut océanographique, sculpture monumentale «La Science découvrant les richesses de l'Océan » à l'occasion des festivités organisées pour le vingtcinquième anniversaire de son règne (11-13 avril 1914), portrait sculpté du Prince, buste du Prince (1927).

\section{Un authentique temple de la science}

\section{Urbanité, architecture et science}

Avant même d'entreprendre son travail, Pontremoli se sait soumis à une triple gageure : faire honneur au mécène qui offre le bâtiment à une science nouvelle, concevoir un programme moderne, pratique et pédagogique et, enfin, résoudre avantageusement l'équation que forme un terrain d'angle, étroit et pentu. Ce dernier point est d'importance et pose un problème général d'urbanité : comment élever une façade, une monumentalité même très contenue, sur le petit côté du terrain? Comment épouser à l'avantage du programme la pente du terrain? Dans sa réponse, l'architecte va choisir de donner de l'ampleur à sa façade principale en privilégiant l'inscription en parallèle sur la voie secondaire tout en bénéficiant, par un adroit recul, de la vision élargie qu'offre l'angle de deux voies.

21 Profitant de cette disposition, l'entrée principale (rue Panhard) devient axiale, surélevée. L'entrée secondaire (boulevard St-Marcel) s'ouvre de plain-pied, facilitant ainsi les transbordements et l'accès au sous-sol (personnel, fournisseurs, collections). Pontremoli accentue la rupture que marque le bâtiment en le calant en creux, sous le niveau du trottoir, usant d'un jeu de cours anglaises éclairant en jour direct le sous-sol et les paillasses des laboratoires. Il rééquilibre l'ensemble en compensant dans sa masse le procédé en contrebas par une élévation de quatre niveaux coiffés de combles à fortes pentes.

Pontremoli conçoit une façade composite horizontale (ill. 1) constituée d'un bâtiment symétrique d'esprit classique à corps central de proportion carrée, calé par deux avantcorps latéraux légèrement saillants. 


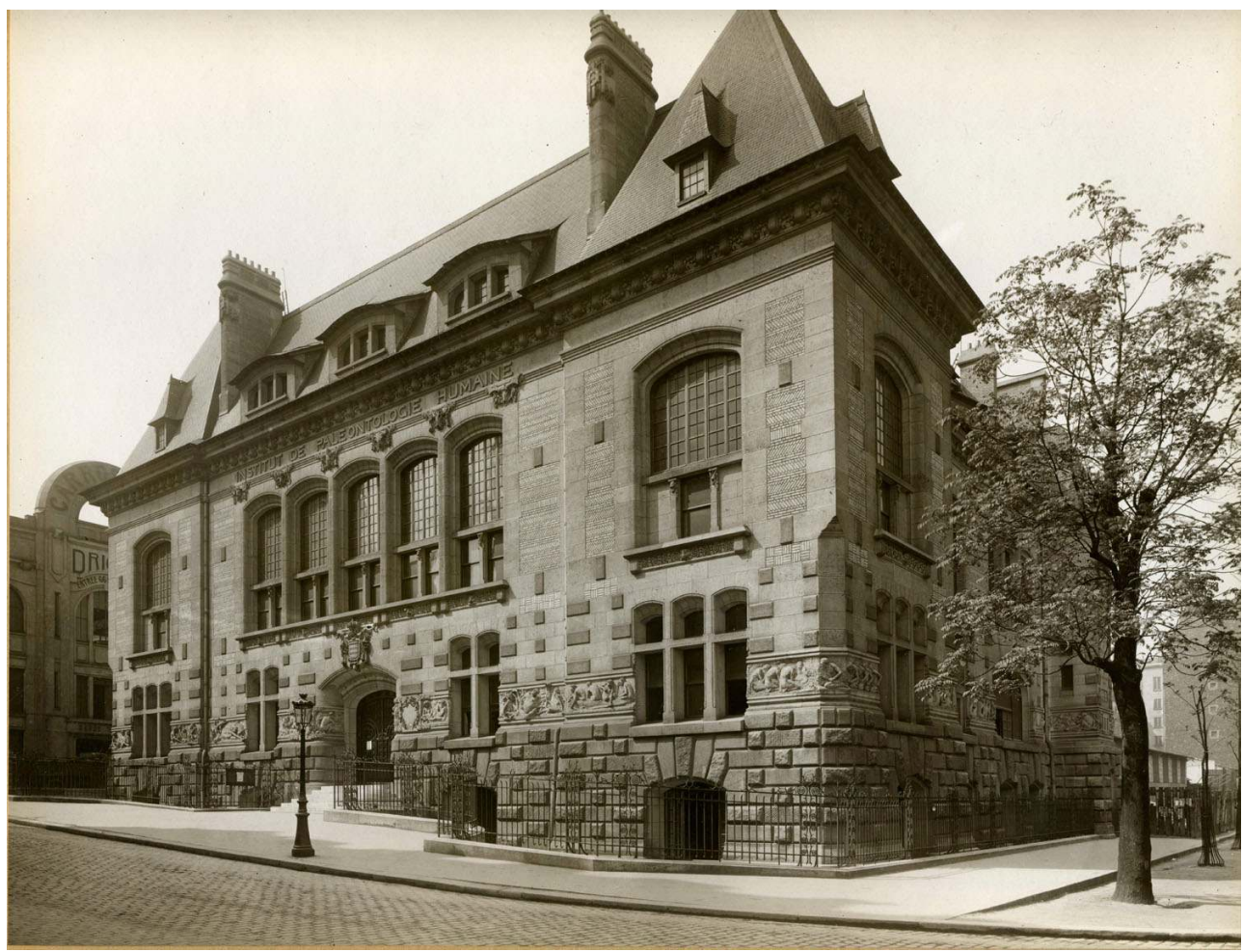

Perspective boulevard Saint-Marcel rue René Panhard

Photo Vizzavona 1920 (c) Fondation I.P.H.

Verticaux eux-mêmes, ils sont en rotation sur les voies latérales. Les éléments sont liés entre eux par une corniche et une imposante toiture percée de lucarnes.

La modénature de la façade gradue un soubassement en pierre d'Euville striée, aux bossages rustiques, parsemé de saillies antiques, sauf sur les chaînes d'angle où il fait place à un calepinage de briques esthétisé.

La quête de la lumière occupe une place majeure et originale dans la composition. La particularité de chaque baie, identiques sur les trois corps se développant verticalement sur deux niveaux d'usage, est l'association d'une verrière à petits-bois prolongeant deux châssis coulissants appuyés sur des appuis filants et des tables sculptées. L'ensemble des ouvertures dominantes dans la maçonnerie s'inscrit sous l'arc surbaissé des linteaux de briques en voussures.

\section{La composition intérieure}

26 Le projet de Boule et Breuil «était sur les locaux, sans aucun esprit de luxe ; mais, sur ce point, le Prince voulut faire honneur à sa haute situation $»^{11}$. Par de nombreux aspects, le programme intérieur renvoie à certains motifs de l'architecture viennoise du début du $\mathrm{XX}^{\mathrm{e}}$ siècle (enroulements du salon des dames au café Heinrich-Off, menuiseries extérieures de la maison Scheu, verrière de la caisse d'épargne de Vienne) et est une espèce d'écho à Kérylos ${ }^{12}$. On peut ainsi distinguer les ensembles menuisés de la salle de conférences, le mobilier dessiné pour l'usager (bureaux, tables, fauteuils, etc.), les ferronneries à base d'enroulements (ill. 2) et les mosaïques qui suggèrent un alphabet 
symbolique primitif (roues solaires, svastikas), les boiseries sculptées d'inspiration polynésienne (comme ces masques de dragons grimaçants qui encadrent l'entrée de la bibliothèque).

\section{2 : Escalier d'honneur de l'Institut de paléontologie humaine}

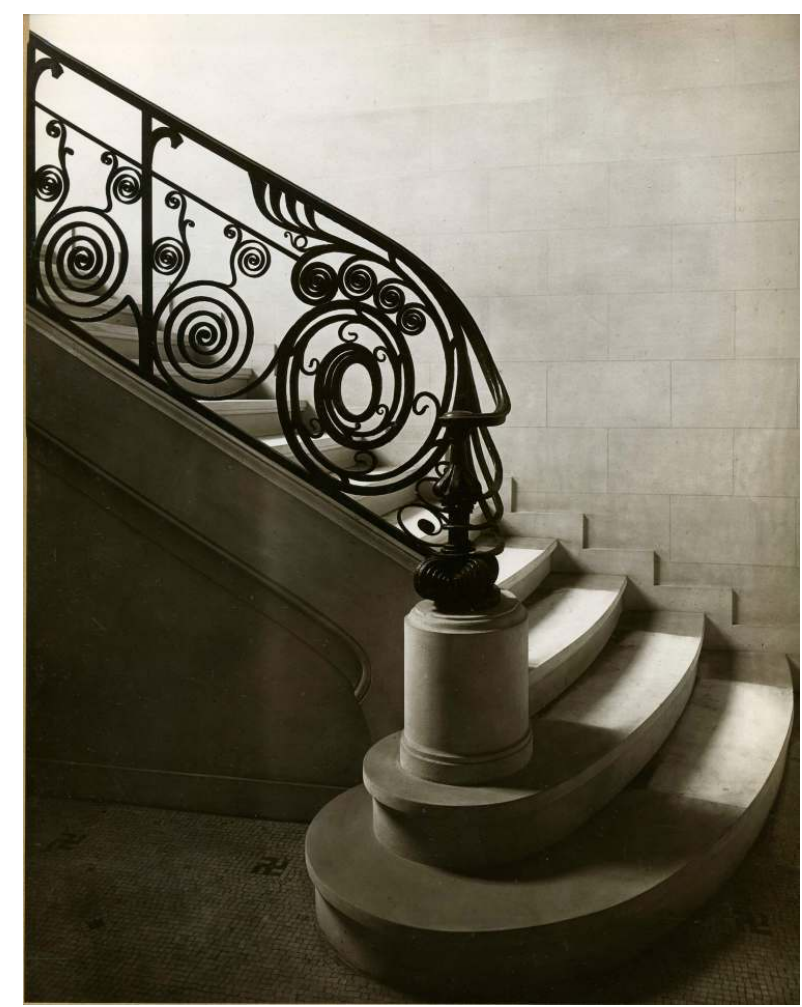

Ferronnerie maître Robert

Photo Vizzavona 1920 @ F Fondation I.P.H.

Deux grandes toiles peintes, réalisées par André Dewanbez (1867-1943) d'après les relevés pariétaux établis par Breuil à Altamira, complètent ce programme (ill. 3). 
III. 3 : Couloir au rez-de-chaussée de l'Institut de paléontologie humaine

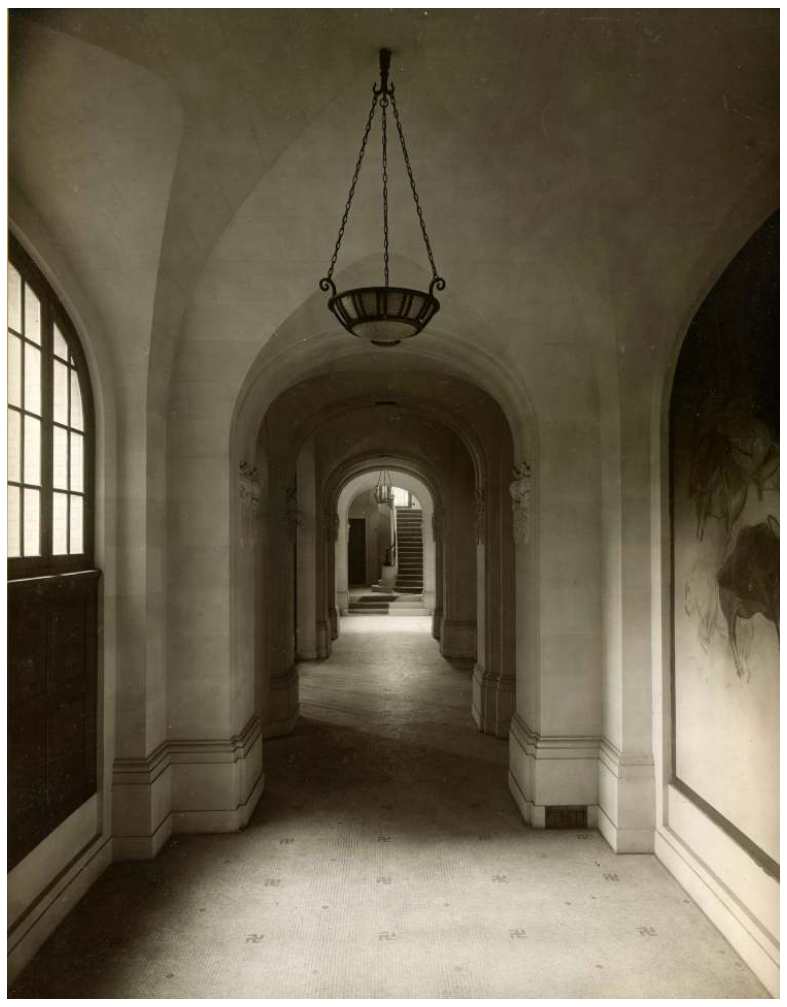

Peintures d'André Devanbez

Photo Vizzavona 1920 ㄷ Fondation I.P.H.

L'édifice comprend au sous-sol les ateliers de préparation des collections et de moulage, les salles de déballage et de classement provisoire des fouilles; au rez-de-chaussée, une grande salle de conférences et d'expositions, les bureaux du directeur et son secrétariat, des laboratoires (photographie, chimie), les cabinets de travail des professeurs; au premier étage, trois salles de collections de comparaison correspondant aux chaires d'enseignement, une bibliothèque, des cabinets de travail réservés aux chercheurs effectuant un séjour temporaire à l'institut; au deuxième étage, des bureaux et des réserves; au troisième étage, une salle de dessin et les dépôts des publications.

L'amphithéâtre (ill. 4) est conçu selon un plan carré à double volume et mezzanine extérieure. 
III. 4 : Salle de conférence de l'Institut de paléontologie humaine

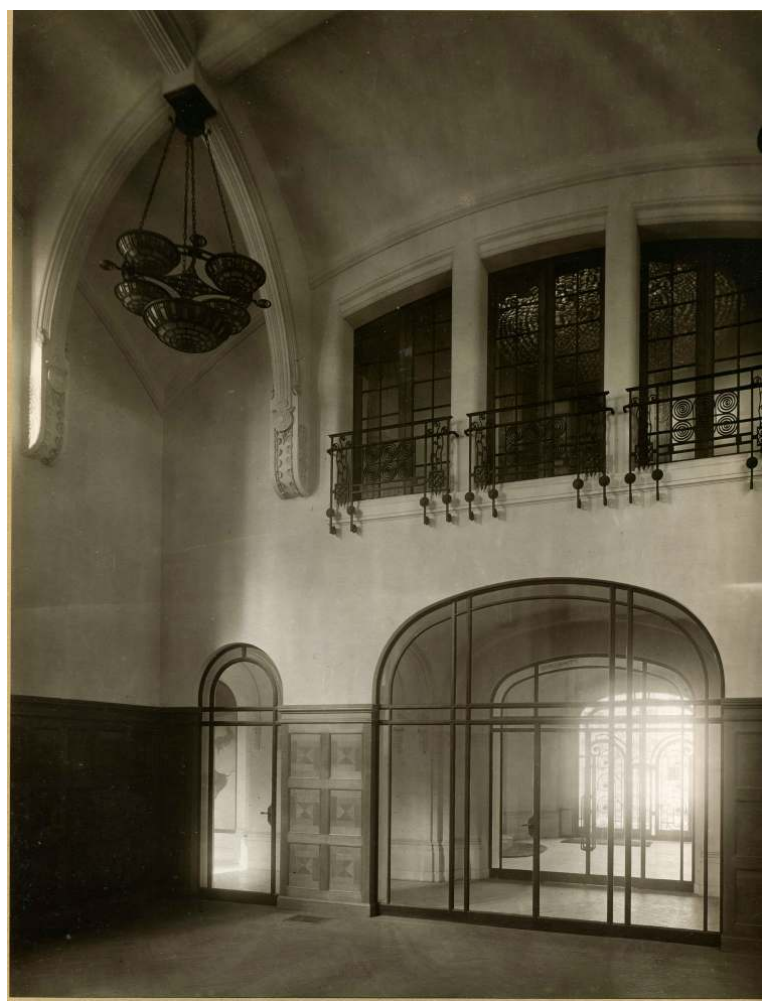

Photo Vizzavona 1920 ( ) Fondation I.P.H.)

L'éclairage naturel est assuré par une coupole vitrée à pavés de verre ronds et deux panneaux vitrés à meneaux en pierre composés de verrières hautes et châssis ouvrants verticaux. La voûte à contreforts croisés est surbaissée pour libérer la portance de la coupole. La décoration se compose de lambris à corniche ronde, d'un panneautage carré à lames dynamiques, d'une estrade de présentation, d'un tableau noir à cadre sculpté, de lustres d'éclairage électrique à cinq demi-globes, d'un mobilier (bureau, table, bancs) en chêne à structure affichée. La mezzanine à panneau vitré coulissant sert de poste de projection (photographique et cinématographique) et offre un point de vue sur la salle pour les retardataires et ceux qui n'ont pu y prendre place.

Les salles de collections de paléontologie humaine et d'anatomie comparée, de volume carré, sont disposées aux angles du bâtiment sur une double hauteur avec mezzanine, avec escalier d'accès à quartier tournant en niche. Éclairées par des verrières, des châssis coulissants bas et des lustres à quatre demi-globes en serrurerie et verre dépoli, elles mettent en valeur des vitrines menuisées à tiroirs pour la présentation des pièces. On retrouve celles-ci dans la salle d'art mobilier et d'industries lithiques (avec ajout de deux compositions d'angle à trois plans), dite « salle Breuil ».

La bibliothèque (ill. 5) est de volume rectangulaire à double hauteur, avec mezzanine de la longueur du corps principal, à trois côtés fermés d'étagères pour livres et boîtes à tirés à part. 


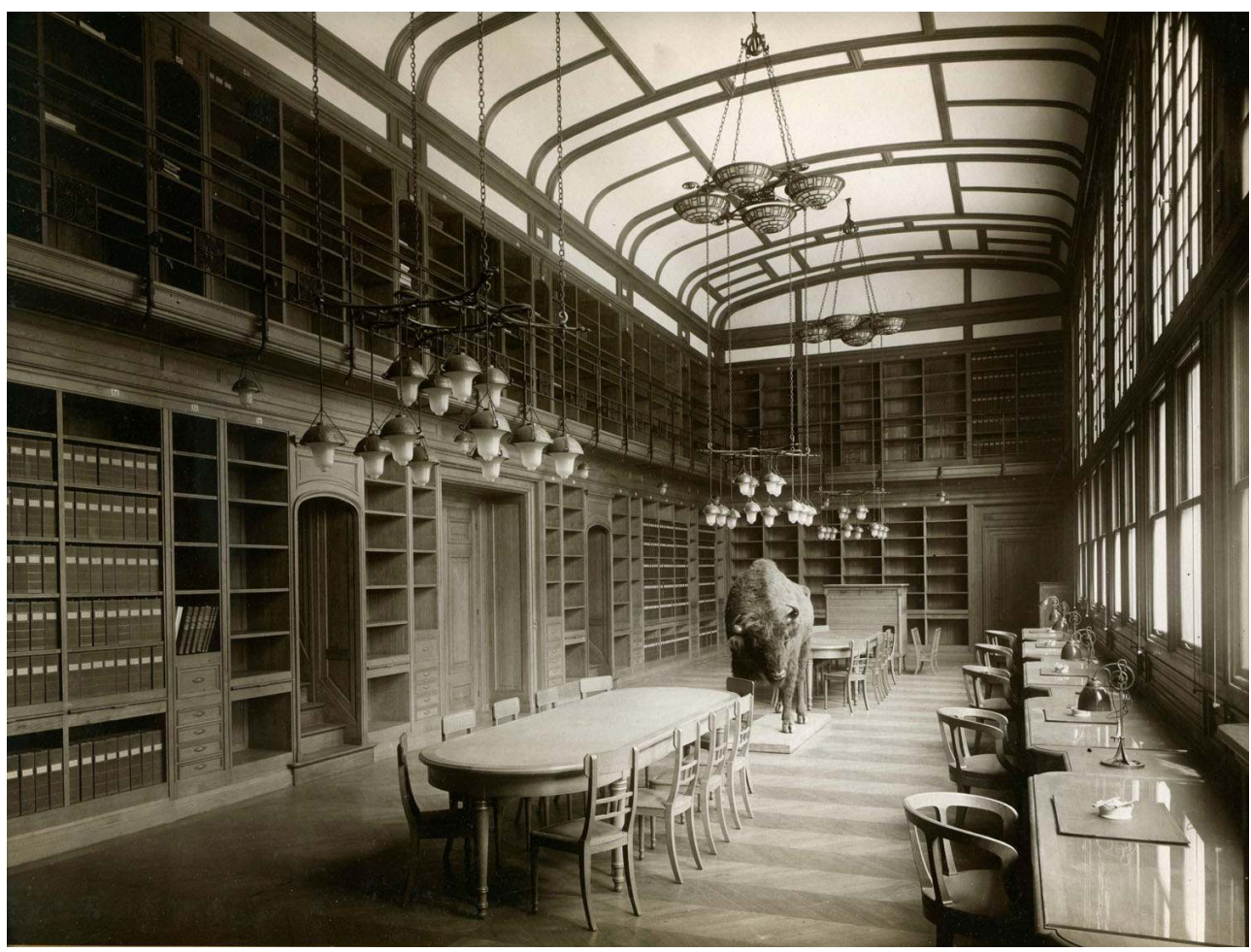

Bison d'Europe naturalisé, souvenir de la chasse du prince Albert ler à Bialowieza

Photo Vizzavona 1920 (c) Fondation I.P.H.

L'ensemble est face à la lumière dispensée par une verrière à cinq hautes fenêtres à quatre vantaux sur châssis coulissants à l'anglaise. La menuiserie est en chêne avec plancher en parquetage en point de Hongrie. Un double escalier en niche permet l'accès à la mezzanine qui s'inscrit dans l'épaisseur de la façade longitudinale. Le plafond surbaissé est rythmé par des caissons à moulures en bois et divisé selon la structure porteuse de l'édifice unifiant les deux longs pans.

\section{Une architecture illustrée}

Dessiné sur plans carrés, l'ensemble architectural est marqué par une forte unité de composition. Simple, élégant, sans sécheresse, il est frappé, comme l'est une monnaie, par la frise en bas-relief se déroulant à hauteur de vue sur les trois façades.

Dans les plans originels, Pontremoli a esquissé des groupes de singes, des scènes de chasse, des corps à corps épiques entre l'homme et des animaux sauvages (aurochs, lion, crocodile, etc.) et même un mammouth. Autant de partis pris artistiques conformes aux représentations de l'époque qui placent l'homme des "âges farouches» dans une ambiance intensément tragique, souvent dans des situations violentes. On le voit alors aux prises avec des bêtes, plus terribles les unes que les autres, au cours de chasses ou de combats pour sa survie. Ainsi, nombre des sculptures offertes au promeneur du Jardin des plantes illustrent cette règle du genre. Le drame n'est jamais loin.

Boule et l'abbé Breuil vont réorienter l'ensemble dans une optique sensiblement moins onirique, en l'adaptant aux recherches qui seront menées dans l'institut: une histoire 
naturelle de l'homme fossile associant faits de culture et faits de nature, collecte des matériaux et analyse. Le travail confié à Roux évoquera donc les hommes des temps préhistoriques grâce aux plus récentes découvertes de l'archéologie et, usant du comparatisme, éclairera l'histoire de l'homme au travers des «derniers reliquats des humanités primitives» (Australiens, Fuégiens, Négritos), les derniers chasseurscueilleurs.

Ce qui frappe dans sa sculpture, c'est l'harmonie générale qui s'en dégage, tant en termes d'exécution que de choix des motifs et figures. Les personnages des scènes ethnographiques ne sont jamais mis dans une situation qui pourrait paraître ridicule ou dépréciative. L'ensemble se veut explicitement descriptif et met en valeur la quotidienneté des actes des groupes humains, c'est-à-dire à travers ce qui pourrait représenter autant de "points communs $»^{13}$ avec les pratiques des hommes préhistoriques. La question de l'homme primitif n'est pas un problème à résoudre sur le plan anatomique, mais un fait culturel, cultuel, civilisationnel.

L'ensemble est apaisé, exempt de toute sauvagerie inutile. Il en est ainsi, par exemple, de la chasse. Elle est évoquée à deux reprises : comme technique (chasse masquée au bison) et comme métaphore de la domination de l'homme sur la nature. Dans ce dernier cas, l'artiste a représenté le cadavre d'un gorille, vaincu, exhibé aux yeux de tous, agacé du bout d'une herbe par deux jeunes Africaines, sans doute enhardies par les entraves qui lient encore la bête tuée. L'homme n'est pas opposé à la nature. Il la respecte et la craint à la fois. Il compose et semble en équilibre avec elle.

$\mathrm{Au}$ total, Roux réalise dix-sept scènes et plusieurs éléments de détail (crânes, objets d'art mobilier préhistorique, pièces lithiques et osseuses, etc.). Les " Australiens » y occupent une place majeure, Roux s'inspirant d'illustrations tirées d'ouvrages de Baldwin Spencer (1860-1929) et Francis James Gillen (1855-1912). Les usages culturels (« arc musical», feu, navigation, pêche, outils, tatouages, pratiques de subsistance) et cultuels (scènes funéraires, totem) les plus variés sont mis en avant ${ }^{14}:$ «La musique primitive : l'arc musical des peuples nègres ", "Gorille tué et négresses ", «Les origines de la navigation et de la pêche avec l'arc, chez les Négritos des îles Andaman", "Scène funéraire australienne ", "Les Aruntas (Australiens) en train de peindre sur un tertre allongé le totem serpent Wollunqua, afin que le gibier se multiplie», "Australien polissant une hache ", "Groupe d'Australiens - Celui de gauche décore d'incisions le dos de son compagnon ; les autres taillent et emmanchent des instruments de pierre taillée » (ill. 6), "Deux Australiens faisant du feu par friction de deux morceaux de bois", "Chimpanzés ", "Eskimo faisant des incantations sur une tombe marquée de bois de Renne ", "Eskimo dépeçant un Élan - À côté de lui, les chiens, le premier animal domestique ", "Campement fuégien: hutte primitive, cuisine entre des pierres », «Les origines de la céramique, du moulin à écraser le grain et du tissage chez les nègres », « La chasse masquée chez les peaux-rouges » pour laquelle il s'inspire d'une des peintures de George Catlin (1796-1872). 
III. 6 : « Groupe d'Australiens - Celui de gauche décore d'incisions le dos de son compagnon ; les autres taillent et emmanchent des instruments de pierre taillée »

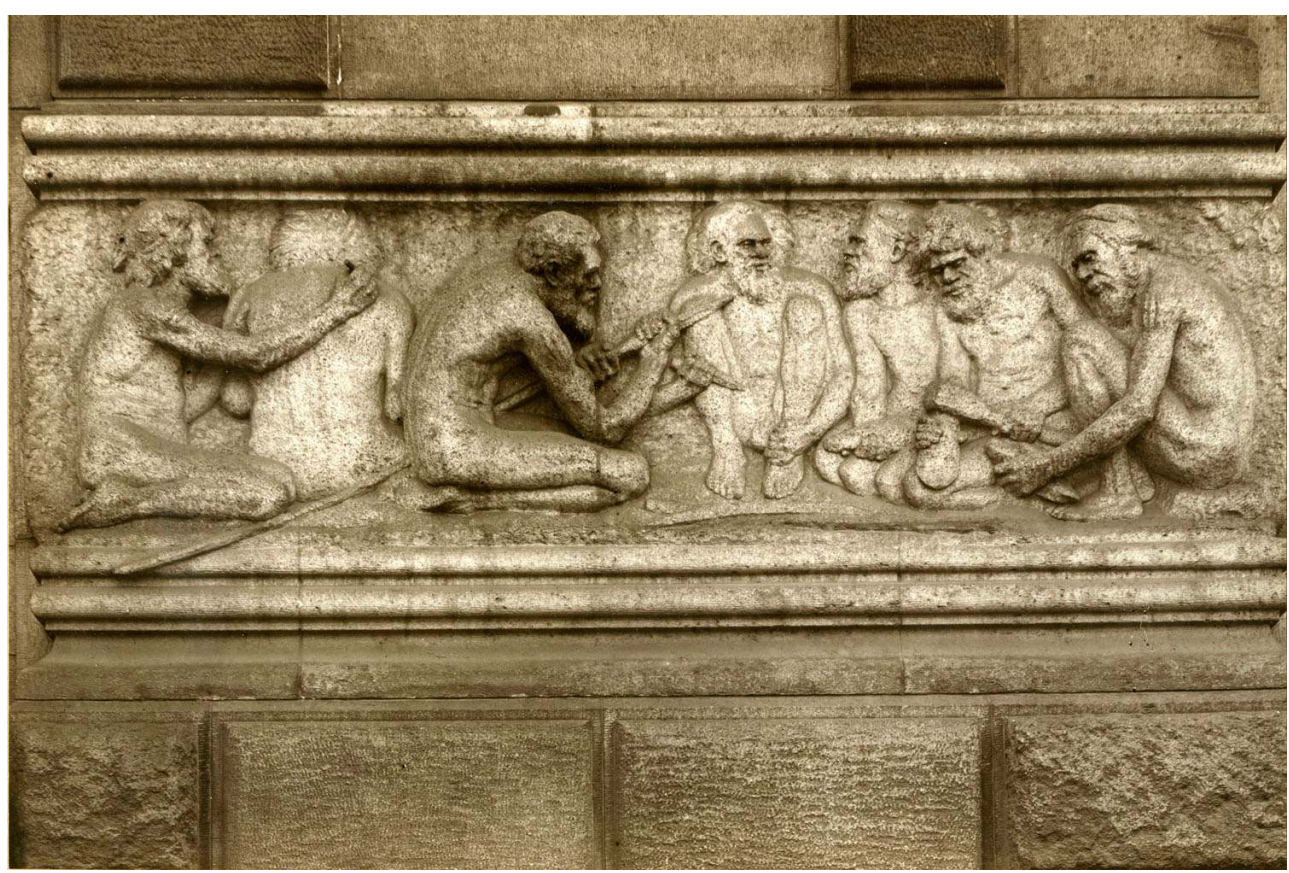

Photo Vizzavona 1920 @ Fondation I.P.H.

40 Quelques scènes illustrent directement la préhistoire et les travaux notables des chercheurs de l'institut (paléoanthropologie, art du paléolithique supérieur). Deux hommes modernes (Homo sapiens) encadrent l'entrée principale et dévoilent la richesse de leur civilisation passée et l'étendue de leur expression artistique naturaliste. Un «aurignacien » sculpte la Vénus à la corne de Laussel et, face à lui, un «magdalénien » peint un Bison de la caverne de Font-de-Gaume (ill. 7). 
III. 7 : « L'homme de l'Âge du Renne sculpte et peint un Bison de la caverne de Font-de-Gaume »

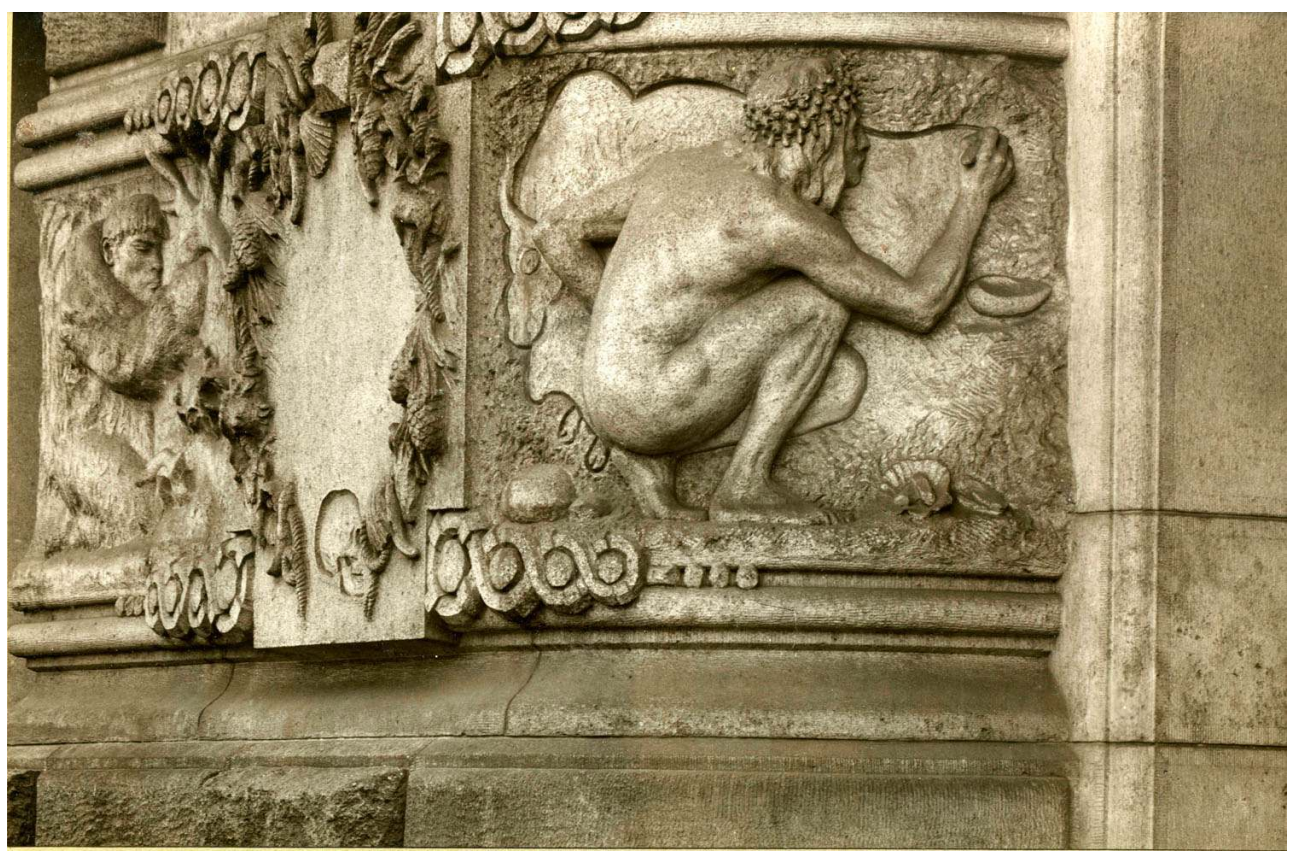

Constant Roux

Photo Vizzavona 1920 (c) Fondation I.P.H.

41 À ses pieds, les outils de l'artiste (ocre, silex pour les gravures, broyeur). À proximité, une des «Vénus » de Grimaldi, des colliers de coquillages, le Mammouth en bois de renne et ronde-bosse de Bruniquel, etc. Par contraste, tenu à l'écart, au-dessus de l'entrée de service, associé aux grands singes, isolé dans sa grotte, taillant grossièrement une pierre, le néandertalien de la Chapelle-aux-Saints semble se terrer.

42 Jusqu'à la seconde guerre mondiale, l'I.P.H. aura été le seul centre de recherches et d'enseignement en France entièrement dédié à l'étude de l'homme préhistorique. Point d'ancrage pour des chercheurs et moteur d'une nouvelle dynamique scientifique, il est également une réussite artistique, saluée et récompensée ${ }^{15}$. Il représente dans une certaine mesure autant d'instantanés d'une science, de ses connaissances, voire de ses « a priori ». Plus généralement, il illustre la capacité de Pontremoli à reprendre pleinement à son compte les attentes de son commanditaire et les contraintes d'usage spécifiques de l'édifice. Sans doute s'agit-il là de cette part du monument qui ne meurt pas, de ce « quelque chose d'immortel $»^{16}$.

\section{NOTES}

1. Les abbés Henri Breuil (1877-1942) pour l'ethnographie préhistorique, Hugo Obermaier (1877-1946) pour la géologie appliquée à la préhistoire, le professeur du Muséum national d'histoire naturelle René Verneau (1852-1938) pour l'anthropologie. Sur l'abbé Breuil, 
voir Frédéric Chappey dir., Sur les chemins de la Préhistoire. L'abbé Breuil du Périgord à l'Afrique du Sud, Paris, Somogy éditions d'art, 2006, 223 p.

2. En attendant, le personnel avait trouvé refuge à l'Institut océanographique rue SaintJacques.

3. Dominique Jarrassé, «Emmanuel-Élisée Pontremoli, architecte, directeur de l'École nationale supérieure des beaux-arts ", Archives juives, 1997, n 30/2, p. 125-127.

4. Emmanuel Pontremoli, Propos d'un solitaire. S.l., n.d., p. 70.

5. Ibid., p. 104.

6. Ibid., p. 104.

7. Ibid., p. 105.

8. Ibid., p. 106.

9. Ibid.

10. Laurent Noet, «Constant Roux (1865-1942), un sculpteur à la cour du prince Albert $\mathrm{I}^{\mathrm{er}} \mathrm{de}$ Monaco ", Annales monégasques, 2003, n²7, p. 161-190.

11. Henri Breuil, «Souvenirs sur le prince Albert de Monaco et son œuvre préhistorique ", Bulletin de la société préhistorique de France, 1951, t. 48, p. 288.

12. Pontremoli réemploie à l'I.P.H. certains dessins faits pour Kérylos et fait appel à plusieurs artisans qui ont travaillé là-bas.

13. Henri Breuil, "Les plus anciennes races humaines connues", Revue des sciences philosophiques et théologiques, 1909, p. 710-758.

14. Les titres ci-après sont ceux alors établis par Breuil.

15. Prix Lheureux de la Ville de Paris à Pontremoli (1913) puis Roux (1917), prix Berger de l'Académie des beaux-arts obtenu conjointement (1920).

16. Georges Gromort cité par Jean-Louis Cohen (dir.), Les Années 30, l'architecture et les arts de l' espace entre industrie et nostalgie, Paris, éditions du patrimoine, 1997, p. 222.

\section{RÉSUMÉS}

Plusieurs points caractéristiques de l'histoire de l'architecture du début du $\mathrm{XX}^{\mathrm{e}}$ siècle convergent pour conférer à l'édifice de l'Institut de paléontologie humaine conçu par Emmanuel Pontremoli (1865-1956) son intérêt particulier. L'observation méthodologique met en exergue trois lectures imbriquées : celle du commanditaire, celle du programme spécifique et celle de l'architecture symbolique d'une période. Cet institut illustre une étape du développement des études préhistoriques (de la curiosité savante à la régulation institutionnelle) fondé sur un mécénat actif. La conception et la réalisation de l'établissement scientifique sont marquées par la collaboration de deux artistes renommés associés sur la base d'un programme précis. La conjugaison de la composition générale de l'édifice, son langage architectural et l'influence fonctionnelle en font un témoin d'une période qui porte la marque d'une lente transformation culturelle bouleversant le «milieu» des Beaux-Arts, alors partagé entre éclectisme et mouvement moderne, dont le médiateur sera Pontremoli comme directeur (1934-1938) et chef d'atelier. 
Several characteristics of early $20^{\text {th }}$ century architectural history converge to give the Institute for Human Paleontology designed by Emmanuel Pontremoli (1865-1956) a particular interest. Three interlinked points of view are brought out by methodological observation, whether speaking of the one commissioning, the specific planning or the contemporary architecture. This institute illustrates one stage in the development of prehistoric studies (from scholar curiosity to institutional control) based on an active sponsorship. The design and the construction of this scientific establishment are marked by the collaboration of two famous artists who started working together from a precise program. Regarding the general structure of the building, the architectural language and the functional influence, the building testifies of a period of slow cultural transformation changing completely the Beaux-Arts circle, at that time torn between Eclecticism and Modern style. With this matter, Pontremoli will act as a mediator, as a headmaster (1934-1938) and as a school teacher in the Ecole des Beaux-Arts.

Das von Emmanuel Pontremoli (1865-1956) konzipierte Pariser Institut für Paläontologie des Menschen bietet in mancher Hinsicht ein hervorragendes Beispiel für die Geschichte der Architektur des frühen 20.Jahrhunderts. Die methodologische Untersuchung des Gebäudes bietet drei aufeinanderwirkende Leseebenen: Rolle des Auftraggebers, spezifische Eigenschaften des Bauprogramms, architektonische Formen als Spiegelbid der Zeit. Die Gründung des Instituts, von aktivem Mäzenatentum unterstützt, entspricht einer neuen Entwicklungsphase in der vorgeschichtlichen Forschung, zwar deren Übergang von gelehrter Neugierde zu institutioneller Regelung. An der Überarbeitung und der Fertigstellung des wissentschaftlichen Institutsbaus beteiligen sich zwei berühmte Künstler, die gemeinsam an dem festgestellten Programm mitwirken. Die Gesamtauffassung des Gebäudes, dessen architektonische Formensprache, sowie die funktionalistische Orientierung des Ganzen führen die langsamen kulturellen Änderungen vor Augen, die das «Milieu» der École des Beaux-arts, geteilt zwischen Eklektizismus und Moderne, ins Wanken bringen. Pontremoli, von 1934 bis 1938 als Direktor, dann als Atelierchef, übernimmt die Vermittlerrolle in dieser Umgestaltung.

\section{AUTEURS}

\section{ARNAUD HUREL}

Arnaud Hurel est historien, docteur en histoire contemporaine (Paris IV Sorbonne), ingénieur au département de préhistoire du Muséum national d'histoire naturelle. Ses recherches portent sur la mise en perspective de la pensée en archéologie préhistorique à travers une approche prosopographique confrontée à l'histoire des idées, de la Révolution à la seconde guerre mondiale. Ce travail concerne quatre domaines essentiels : les enjeux des questionnements paléoanthropologiques, la question des préhistoriens catholiques entre les deux guerres mondiales, la reconnaissance du patrimoine archéologique préhistorique et la dimension institutionnelle de ces problématiques. Il est l'auteur de nombreux articles scientifiques et de vulgarisation consacrés à l'histoire du patrimoine et de l'archéologie préhistoriques et, en collaboration avec Amélie Vialet, de Teilhard de Chardin en Chine. Correspondance inédite (1923-1940), Éditions du Muséum-Édisud, 2004. Adresse électronique : hurel@mnhr.fr

\section{ALAIN DUBOURG}

Alain Dubourg est architecte et méthodologue. Ses travaux portent sur des interrogations essentielles : comment travaillent les architectes? dans quel but? à l'usage de qui ? la passion d'architecture restant substruction. Ainsi, après des années de pratiques individuelles ou associatives, le besoin d'analyse logique des champs d'influence l'a amené à s'intéresser à cette 
autre discipline, tout aussi enthousiasmante, qu'est la méthodologie, c'est-à-dire comprendre pour créer. Il est l'auteur, en collaboration avec Christophe Gobin et Jean-Luc Salagnac, d' Optimisation de la filière béton en habitat collectif : approche fonctionnelle (CIM béton, 2001), et de Pratique d'analyse méthodologique (AFAV, 2000). Il est, par ailleurs, secrétaire-fondateur de l'association « De l'œuvre à sa mémoire » qui milite en faveur d'une approche pédagogique et partagée de l'architecture. 